\title{
Probiotics maintain intestinal secretory immunoglobulin A levels in healthy formula-fed infants: a randomised, double-blind, placebo-controlled study
}

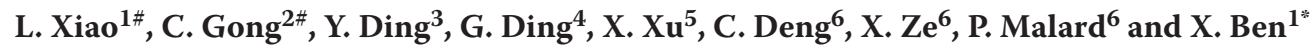 \\ ${ }^{1}$ Department of Neonatology, Shanghai First Maternity and Infant Hospital, Tongji University School of Medicine, Shanghai \\ 201204, China P.R.; ${ }^{2}$ Department of Pediatrics, Shanghai East Hospital, Tongji University School of Medicine, Shanghai \\ 201204, China P.R.; ${ }^{3}$ Department of Neonatology, First People's Hospital of Zhangjiagang, Soochow University School of \\ Medicine, Jiangsu 215600, China P.R.; ${ }^{4}$ Department of Respiratory Medicine, Shanghai Children's Hospital, Shanghai \\ Jiaotong University School of Medicine, Shanghai 200040, China P.R.; ${ }^{5}$ Lallemand Health Solutions Inc., 6100 Avenue \\ Royalmount, Montreal, QC H4P 2R2, Canada; ${ }^{6}$ Biostime (Guangzhou) Health Products Ltd., 187 Lianguang Rd, East \\ District, Economic and Technological Development District Guangzhou, China P.R.; \#these authors contributed equally \\ to thiswork; benxm@163.com
}

Received: 8 February 2019 / Accepted: 19 April 2019

(c) 2019 Wageningen Academic Publishers

\section{OPEN ACCESS @(1) (0) RESEARCH ARTICLE}

\begin{abstract}
Formula-fed infants are more susceptible to infectious diseases because they lack the maternal immune factors transferred from breast milk, while their own immune system is still immature. As timely probiotic administration was suggested to promote immune system development in formula-fed infants, this study aimed at assessing the safety and the effects of a probiotic supplement (Bifidobacterium infantis R0033, Bifidobacterium bifidum R0071, and Lactobacillus helveticus R0052) on mucosal immune competence and digestive function in formula-fed infants. Healthy infants (3.5-6 months old) were randomised to receive either probiotic- $(\mathrm{n}=66)$ or placebo-supplemented $(\mathrm{n}=66)$ formula once a day for four weeks. In the probiotics group, faecal secretory immunoglobulin A (SIgA) levels remained similar between visit 2 (baseline; V2) and visit 3 (end-of-treatment; V3), but decreased in the placebo group. Changes in SIgA levels following treatment $\left(\log _{10} \Delta \mathrm{V} 3-\mathrm{V} 2[95 \% \mathrm{CI}]\right)$ between the probiotic and placebo groups were statistically significant ( $23 \mathrm{ng} / \mathrm{dl}[-57 ; 102]$ and $-137 \mathrm{ng} / \mathrm{dl}[-212 ;-62]$, respectively $(P=0.0044$; ANCOVA)). While $\log _{10} \Delta \mathrm{V} 3-\mathrm{V} 2[95 \% \mathrm{CI}]$ for salivary SIgA levels increased in both groups, this trend was more pronounced in the probiotics than in the placebo group with an increase of $123 \mathrm{ng} / \mathrm{dl}[9 ; 236]$ and $37 \mathrm{ng} / \mathrm{dL}$ [-72;147], respectively $(P=0.2829 ;$ ANCOVA $)$. The weekly average number of stools/day was significantly higher in the probiotics group compared to placebo during the last week of treatment for the per protocol population. There was no difference in microbiota composition or anthropometric parameters between groups. No serious adverse event was reported, and all adverse events were mild and unrelated to the product or study. Our results show that formula-fed infants receiving probiotics maintained higher faecal SIgA levels at the end of the four-week treatment period, suggesting a positive effect of probiotics on SIgA production. This study demonstrates the safety of this probiotic formulation in infants. Formula-fed infants may benefit from probiotics supplementation to sustain the development of mucosal immunity.
\end{abstract}

Keywords: probiotic strains, bifidobacteria, paediatric population, immune competence, SIgA

\section{Introduction}

The first months of life are key for establishment of infants' intestinal microbiota. The gut microbiota profile evolves continuously over the first five years of life, until it reaches an adult-like composition (Rodríguez et al., 2015). In breastfed babies, the initial microbiota is composed mainly of aerobic bacteria during the first days of life, with a subsequent dominance of strictly anaerobic bifidobacteria species. Formula-feeding, among other factors, alters 
normal microbiota development. Formula-fed babies display an increased microbial diversity characterised by an enrichment in anaerobic bacteria, such as Bacterioides and Clostridium, with an overrepresentation of Clostridium difficile (Yatsunenko et al., 2012). In addition, protective bifidobacteria species are underrepresented in formula-fed infants compared to breastfed infants (Lee et al., 2015). For example, breastmilk oligosaccharides were shown to foster colonisation by specific Bifidobacterium strains, notably Bifidobacterium longum subsp. infantis (B. infantis) (Lee et al., 2015). Now that recent milk formulas are formulated with a $\mathrm{pH}$ closer to that of breast milk and include nondigestible oligosaccharides (as prebiotics), they partially mimic the effect breast milk and improve colonisation by bifidobacteria (Giovannini et al., 2014; Matsuki et al., 2016; Simeoni et al., 2016). Consistent with this, a number of studies have recently reported similar faecal bifidobacteria counts, at the genus level without identifying the species, in babies fed with breastmilk or formula containing prebiotic oligosaccharides (Penders et al., 2005; Wang et al., 2015).

In addition to its role in defining the early microbiota composition, breastfeeding has been shown to transfer a variety of biologically active factors such as immunoglobulins from the mother, thereby promoting immune system development in newborns (Cacho and Lawrence, 2017). Secretory immunoglobulin A (SIgA), which represents $90 \%$ of the immunoglobulins present in breast milk, protects the intestinal mucosa and prevents infections primarily by promoting pathogens' agglutination in mucus (Cacho and Lawrence, 2017). This crucial firstline of defence against pathogens is lacking in the immature immune system of formula-fed newborns until endogenous SIgA production begins (Lewis and Wilson, 2011). A complex dynamic exists between microbiota composition and SIgA levels in the intestines (Wopereis et al., 2014). Initial colonisation of the newborns' gastrointestinal tract facilitates the development of the gut-associated lymphoid tissue, which initiates endogenous SIgA production (Gensollen et al., 2016). In turn, intestinal SIgA has been shown to maintain a controlled microbial colonisation while preserving mucosal homeostasis (Kato et al., 2014; Mantis et al., 2011). Intestinal SIgA is produced in the gut-associated lymphoid tissue by plasma cells, which are Ig-secreting immune cells differentiated from the B-cell lineage. For the first week and a half after birth, these cells are absent from the newborns' intestinal lamina propria. Endogenous SIgA production by the newborns' plasma cells begins approximately three weeks later (Jakaitis and Denning, 2014). At one month of age, faecal SIgA levels in breastfed infants also include maternal SIgA, and are approximately 18 times higher than in formula-fed infants, supporting the notion that breastfed infants are better protected against infections than their formula-fed counterparts (Guaraldi and Salvatori, 2012).
Considering the significant public health concern resulting from the higher propensity to infections and related lifelong health issues associated with the lower SIgA levels in formula-fed infants, the World Health Organization has recently reiterated its recommendation for exclusive breastfeeding in the first 6 months after birth, and extended the recommendation for mothers to continue providing breastmilk in their infants' diet until two years after birth (Guaraldi and Salvatori, 2012; WHO, 2018a). However, in some cases, breastfeeding may not represent a possible or suitable option. Therefore, easily applicable strategies aimed at supporting immune system maturation, SIgA production, and microbiota establishment in formula-fed infants are needed. Probiotic supplementation in infants has been suggested to sustain immune development and microbiota establishment (Stojkovic et al., 2016) and some probiotic formulations were shown to decrease the risk of necrotizing enterocolitis and sepsis in preterm infants, although the extent of beneficial effects appears to vary according to the formulations used or the premature infants' birthweight (Deshpande et al., 2017; Jacobs et al., 2013; Thomas et al., 2017; Zhang et al., 2016). While studies have yielded promising results following bifidobacteria supplementation in non-preterm infants, even when breastfed (Aloisio et al., 2018), other clinical trials have yielded mixed results and no consensus has been reached regarding the extent of the health benefits of probiotic supplementation in milk formula (Skorka et al., 2017). Here, using a randomised, double-blind, placebo-controlled parallel trial design, we assessed the safety and effects of an oral preparation of probiotics, containing Bifidobacterium infantis R0033, Bifidobacterium bifidum R0071, and Lactobacillus helveticus R0052 on immune and digestive functions in healthy formula-fed infants.

\section{Materials and methods}

\section{Participant selection}

Participants flow through each stage of the study is presented in Figure 1. Healthy infants between 3.5 and 6 months old were recruited for this study. Study eligibility was assessed based on the following inclusion criteria: single birth at gestational age $\geq 37$ weeks (i.e. non-preterm infants (WHO, 2018b)); birth weight >2,500 g; appropriate weight between P20-P80 at the inclusion visit, in accordance with the weight percentile standards for children up to 6 years old in the Shanghai urban areas; formula feeding representing $>80 \%$ of daily food intake at the inclusion visit; absence of gastrointestinal diseases within 1 month prior to the inclusion visit; parents agreement to use one of the recommended infant formulas (i.e. without probiotics, without fructooligosaccharide, with less than $2 \mathrm{~g}$ of galactooligosaccharide/100 g); capacity of the parent or legal tutor to understand the protocol and fill out the 


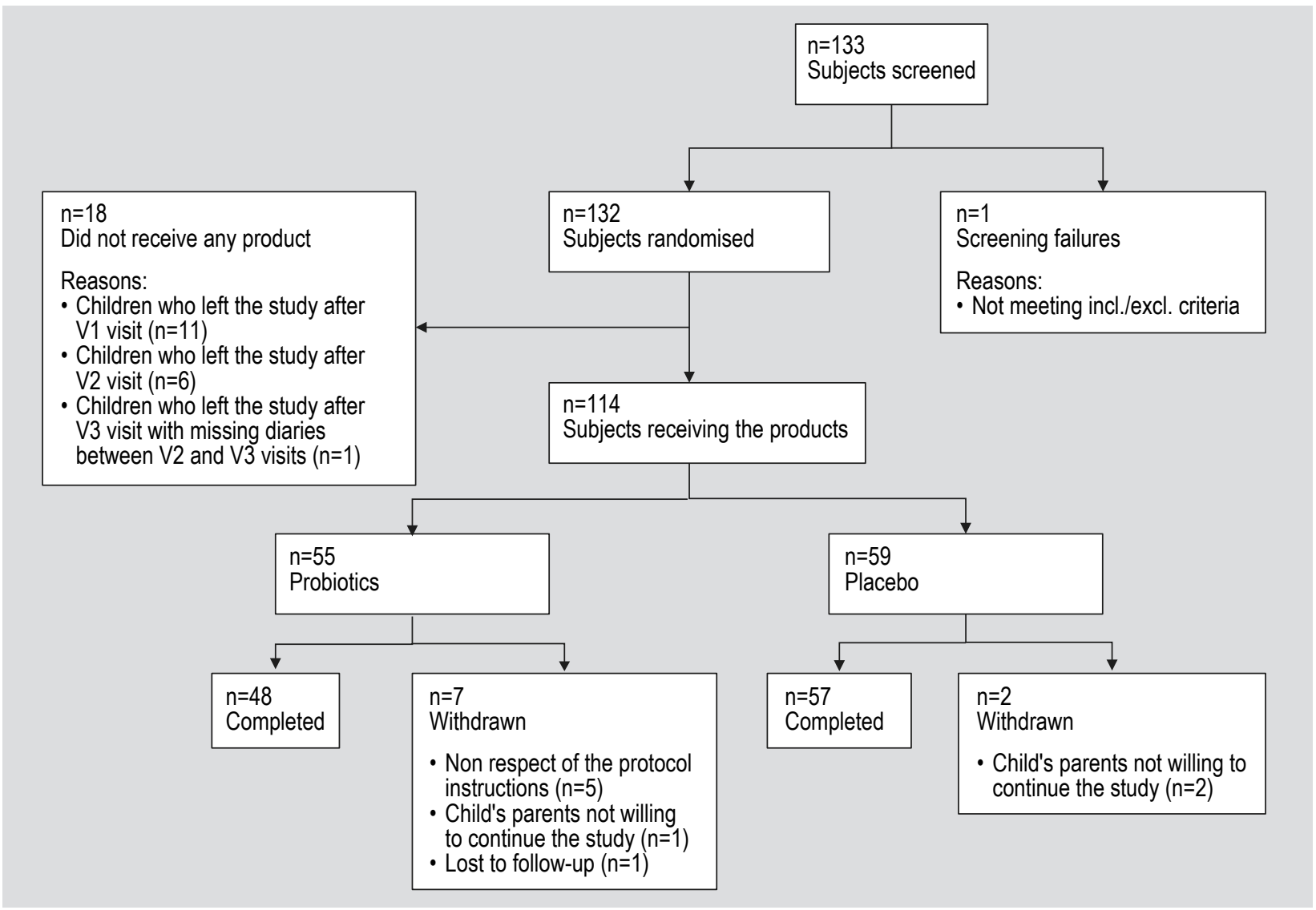

Figure 1. Flowchart depicting the recruitment of participants and providing the reasons for exclusions throughout the study.

infant's diary; consent form signed by at least one of the parents or by the legal tutor properly informed of the study.

However, potential participants meeting one of the following criteria were not eligible for the study: Congenital illness or malformation; significant pre-natal and/or postnatal disease; mothers with metabolic or chronic disease; infants with allergic constitution or known sensitivity to probiotics; infants with serious diseases (e.g. cardiovascular, cerebrovascular, hepatic, renal or hematopoietic system diseases, internal secretion diseases, or mental disorder); infants receiving other drugs during the intervention phase of the study (thereby preventing a clear interpretation of the results and intervention efficacy or influencing data analysis); infants known to have current or previous illnesses/conditions or intervention which could interfere with the study (i.e. impacting tolerance and/or growth), such as gastrointestinal malformations, chronic diarrhoea, malabsorption syndrome, malnutrition, congenital immunodeficiency, major surgery, or other as judged by clinical investigators; infants under oral antibiotic treatment at or within the four weeks preceding the inclusion visit; infants who received any medication or nutritional supplements (including probiotics, or prebiotics except for accepted levels in infant formula) in the four weeks preceding study start; infants who have ever consumed the interventional product; infants who have medical conditions for which a special diet other than standard (non-hydrolysed) cow milk-based infant formula is required (e.g. cow milk allergy, soy protein allergy, fish protein allergy, egg protein allergy, lactose intolerance, galactosaemia); currently participating or having participated in another clinical trial during the month preceding study start; infants whose legal representatives have psychological or linguistic incapability to sign the informed consent form; reasons to presume that parents are unable to meet the study plan requirements (e.g. impossibility to contact study representatives in case of emergency or unstable living conditions).

\section{Ethics and informed consent}

The study was approved by the Medical Ethics Committee of Shanghai First Maternity and Infant Hospital, China. Each parent or legal tutor has signed an informed consent form before infants were enrolled in this study. The study protocol was published previously (Xiao et al., 2017) and the trial was registered in ClinicalTrials.gov (NCT02317406). 


\section{Interventional products composition and dosage}

One probiotics sachet $(1.5 \mathrm{~g})$, which contained $1.425 \times 10^{8}$ cfu of each B. infantis R0033 and B. bifidum R0071, with $9.6 \times 10^{9} \mathrm{cfu}$ of $L$. helveticus R0052, was diluted in the first feeding bottle of the day during four weeks. For the placebo group, an identically packaged, sensorially similar product composed mainly of potato starch was used. Both products were provided by Biostime (Guangzhou) Health Products Ltd.(Guangzhou, China).

\section{Study design and procedures}

A diagram of the study design was published previously (Xiao et al., 2017). This randomised, double-blind, placebocontrolled, two-arm parallel group trial was conducted at the Shanghai First Maternity and Infant Hospital, Shanghai, China. Each participating infant was required to undergo a two-week run-in period before being randomly allocated to receive either the oral probiotic supplement or placebo once daily for four weeks, with a follow-up period of eight weeks. The total duration of the study was 14 weeks, with a total of five study visits. At the first visit, an investigator explained the study procedures to the parent or legal tutor in detail and reviewed the inclusion and exclusion criteria. The investigator also collected relevant demographic information, medical history, and concomitant medications. Study products (probiotics or placebo) were provided to the parents at visit 2 (V2) until the end of the 4 week treatment period at visit $3(\mathrm{~V} 3)$. The infants' diaries were provided to parents or legal tutor at each visit, and they were instructed to fill in these diaries in order to record information such as gastrointestinal symptoms, number of stools/day, compliance and potential concomitant medications. The investigators also conducted a physical examination at each visit, including follow-up visits 4 and 5 , and monitored potential adverse events (AEs).

\section{Saliva sample collection}

Saliva was collected using Salivette ${ }^{\bullet}$ sampling tubes (Sarstedt, Rommelsdorf, Germany). The swab inside of the Salivette tube was held firmly while one end was put in the baby's mouth to be chewed for about $45 \mathrm{~s}$ to stimulate salivation and allow absorption into the swab. The swab with the absorbed saliva was then stored into the Salivette tube, kept at $4{ }^{\circ} \mathrm{C}$ and transported to the laboratory within $24 \mathrm{~h}$ for analysis. Saliva was recovered by centrifuging the Salivette at $1000 \times g$ for $2 \mathrm{~min}$.

\section{Stool sample preparation}

Parents were instructed to collect fresh stool samples in air-tight plastic bags within $24 \mathrm{~h}$ prior to V2 and V3. Stool samples were maintained anaerobically and sent to an external laboratory for analyses. Briefly, stool samples $(0.5 \mathrm{~g})$ were mixed in $5 \mathrm{ml}$ of sterile Ringers solution containing 5\% cysteine and vortexed for 5 to $10 \mathrm{~min}$. Then, the supernatant was geometrically diluted in reagent bottles under sterile conditions.

\section{Secretory immunoglobulin A quantification}

SIgA concentration was assessed using an automated particle-enhanced immunonephelometric assay (Siemens, Munich, Germany) (Booth et al., 2009; Dion et al., 2004; Tzira et al., 2018). Reference curves were generated by multi-point calibration. Serial dilutions of N IgA standard (Siemens) were automatically prepared using a Siemens Specific Protein Analyser and N diluents.

\section{Microbiota composition analysis}

For the faecal microbiota composition analysis, stool samples were prepared in Ringer solution, as described above. Various media, obtained from Qingdao Hai Bo Biotechnology Ltd. (Qingdao, China P.R.) were used for the selective isolation of different microorganisms: freshly prepared De Man, Rogosa and Sharpe (MRS) agar to which mupirocin lithium salt was added, for bifidobacteria; Lactobacillus selection agar supplemented with $1 \mathrm{ml} / 1000$ $\mathrm{ml}$ of Tween 80 and $1.3 \mathrm{ml} / 1000 \mathrm{ml}$ of glacial acetic acid, for lactobacilli; tryptose sulphite cycloserine agar to which 0.8 $\mathrm{ml} / 100 \mathrm{ml}$ of 5\% D-cycloserine was added, for Clostridium perfringens; modified Gifu anaerobic medium agar, to which $80 \mathrm{mg} / \mathrm{ml}$ of vancomycin, $1 \mathrm{mg} / \mathrm{ml}$ of kanamycin, 0.01 $\mathrm{ml} / 100 \mathrm{ml}$ of $1 \%$ vitamin $\mathrm{K} 1,0.25 \mathrm{~g} / 100 \mathrm{ml}$ of hemin, and $7 \mathrm{ml} / 100 \mathrm{ml}$ of blood were added, for Bacteroides; bile esculin azide (BEA) agar, for enterococci; and violet red bile dextrose agar, for Enterobacteria. The seeded, streaked culture plates were placed in anaerobic containers to maintain anaerobic conditions and incubated at $37^{\circ} \mathrm{C}$. Each anaerobic container contained a resazurin oxygen reduction indicator to ensure anaerobicity during incubation. The MRS, Lactobacillus selection agar, and modified Gifu anaerobic medium plates were incubated anaerobically for $48 \mathrm{~h}$; the BEA, tryptose sulphite cycloserine, and violet red bile dextrose agar plates were incubated anaerobically for 24 h. Colony counts were obtained manually and expressed as $\mathrm{cfu} / \mathrm{g}$; numbers over 300 were considered as too numerous to count. After incubation, colonies with representative morphology and colour typical of specific species were randomly selected and identified with the microorganism Mass Spectrometry system (Bruker, Billerica, MA, USA) for confirmation.

\section{Safety assessments}

Anthropometric parameters were documented to assess the effect of the probiotic supplement on infants' growth rate. Occurrence of AEs was monitored throughout the study for product safety assessment. All AEs and serious AEs (SAEs) 
were recorded in the case report forms, whether related to the test product or not. All SAEs were reported as soon as possible to the national food and drug administration (FDA) and local FDA, the sponsor, and infant's hospital.

Descriptive statistics, including the frequency of subjects showing at least one $\mathrm{AE}$, the mean number of $\mathrm{AE}$ for each subject and the number of events, were generated by severity and relationship to the research procedure or study product. The proportion of subjects showing at least one $\mathrm{AE}$ was compared between groups using a chi-square or Fisher Exact test (if the chi-square test is not applicable). The mean number of AE for each subject and the number of events were compared between groups using a Poisson regression.

\section{Statistical analyses}

All statistical analyses were performed by a specialised statistician who remained blinded to study groups. To estimate the efficacy of this trial, both the intention-totreat and per-protocol populations were analysed. Baseline characteristics were compared using either unpaired Student's t-test (parametric test) or Mann-Whitney test (non-parametric test) and a chi-squared or Fisher's exact test (if one of the expected frequencies was $\leq 5$ ). Statistical models were adjusted on breastfeeding history and delivery method. All statistical analyses were performed using the SAS software version 9.3 or higher (SAS Institute Inc., Cary, NC, USA), or the GraphPad Prism software v8.0.2 (GraphPad Software, San Diego, CA, USA). A $P<0.05$ was considered as statistically significant.

\section{Results}

\section{Baseline characteristics of participants}

All analyses were performed on the intent-to-treat (ITT) population. Unless otherwise specified, identical results were obtained using the per-protocol (PP) population. The main demographic parameters of the ITT population, overall $(\mathrm{n}=132)$ and for each study arm, namely placebo $(n=66)$ or probiotics $(n=66)$, are shown in Table 1. No statistical differences between groups were observed at baseline on age $(P=0.7955)$, child's gestational age $(P=0.5200)$, gender $(P=0.6005)$, and breastfeeding history $(P=0.2230)$.

\section{Quantification of salivary secretory immunoglobulin A levels}

There was no statistically significant difference observed on the $\log _{10} \Delta \mathrm{V} 3-\mathrm{V} 2[95 \% \mathrm{CI}]$ of the salivary SIgA concentration between the probiotics and placebo groups (123 ng/dl [9; 236] and $37 \mathrm{ng} / \mathrm{dl}[-72 ; 147]$ respectively; $P=0.2829$ ). However, the increasing trend was more pronounced in the probiotics group compared to the placebo group (Figure 2A).

\section{Quantification of faecal secretory immunoglobulin A levels}

Faecal SIgA concentrations $(\mu \mathrm{g} / \mathrm{dl}$; mean (standard deviation)) were measured at baseline (V2) and at the end of the treatment period (V3) (Table 2). Participants in the probiotics group maintained a higher faecal SIgA concentration over the treatment period compared to those in the placebo group, in whom faecal SIgA levels were significantly reduced at V3 compared to V2 (Figure 2B). Due to strong deviation from assumptions of normality and equal variance (homoscedasticity), analyses were performed

Table 1. Participants baseline characteristics for the intent-to-treat population.

$\begin{array}{lllllc}\text { Endpoint } & \text { Modalities } & \text { Statistical parameter } & \text { Included subjects }(\mathbf{n}=132) & \text { Placebo }(\mathbf{n}=66) & \text { Probiotics ( } \mathbf{n = 6 6 )} \\ \text { Gender } & \text { male } & \mathrm{n}(\%) & 71(53.8) & 34(51.5) & 37(56.1) \\ & \text { female } & \mathrm{n}(\%) & 61(46.2) & 32(48.5) & 29(43.9) \\ \text { Age (days) } & & \text { mean (SD) } & 146.6(24.37) & 147.2(23.56) & 146.1(25.33) \\ \text { Gestational age (days) } & & \text { mean (SD) } & 274.7(5.79) & 274.4(5.67) & 275.0(5.93) \\ \text { Delivery method } & \text { vaginal } & \mathrm{n}(\%) & 56(42.4) & 28(42.4) & 28(42.4) \\ & \text { caesarean } & \mathrm{n}(\%) & 76(57.6) & 38(57.6) & 38(57.6) \\ \text { Was the child ever breastfed? } & \text { yes } & \mathrm{n}(\%) & 65(49.2) & 36(54.5) & 29(43.9) \\ & \text { no } & \mathrm{n}(\%) & 67(50.8) & 30(45.5) & 37(56.1) \\ \text { Is the child currently breastfed? } & \text { yes } & \mathrm{n}(\%) & 0(0.0) & 0(0.0) & 0(0.0) \\ & \text { no } & \mathrm{n}(\%) & 132(100.0) & 66(100.0) & 66(100.0) \\ \text { Was the child ever vaccinated? } & \text { yes } & \mathrm{n}(\%) & 132(100.0) & 66(100.0) & 66(100.0)\end{array}$



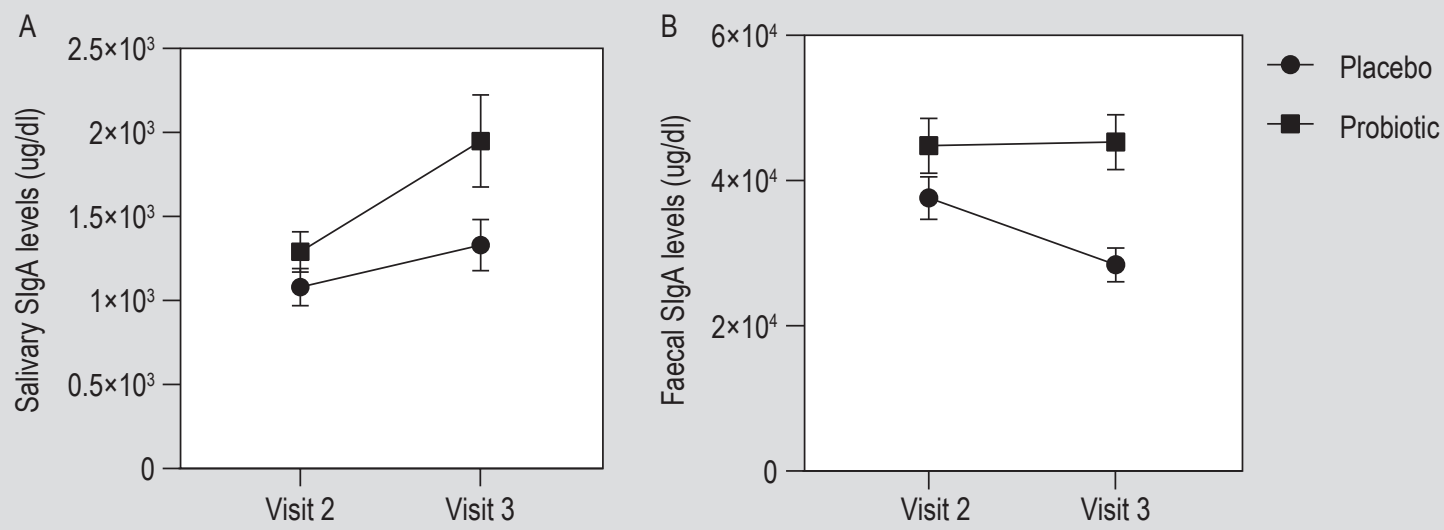

Figure 2. Quantification of secretory immunoglobulin A (SlgA) levels in saliva and faeces at visit 2 and visit 3. (A) Average SlgA concentrations in saliva $(\mu \mathrm{g} / \mathrm{dl})$ for the intent-to-treat (ITT) population in placebo $(n=66)$ and probiotics $(n=66)$ groups at visit 2 and visit 3 . Statistical significance was assessed using the Holm-Sidak method $(\alpha=0.005)$ without assuming a consistent variance $(P=0.096971)$, as well as a non-parametric Student's $t$-test for unequal variance (at $V 3, P=0.04972$ ). (B) Average SIgA concentrations in stool $(\mu \mathrm{g} / \mathrm{dl})$ for the ITT population in the placebo $(n=66)$ and probiotics $(n=66)$ groups at visit 2 and visit 3 . Statistical significance was assessed using the Holm-Sidak method ( $\alpha=0.05$ ), without assuming a consistent variance (at $V 3, P=0.00042$ ).

Table 2. Descriptive statistics of results for faecal secretory immunoglobulin A concentration ( $\mu \mathrm{g} / \mathrm{dl})$ in the intent-to-treat (ITT) population.

$\begin{array}{llccc}\text { Visit } & \text { Statistical parameter }{ }^{1} & \text { ITT population }(n=132) & \text { Placebo }(n=66) & \text { Probiotics }(n=66) \\ \text { V2 } & N \text { (Nmiss) } & 117(15) & 60(6) & 57(9) \\ & \text { mean (SD) } & 4.11 \times 10^{4}\left(2.75 \times 10^{4}\right) & 3.76 \times 10^{4}\left(2.39 \times 10^{4}\right) & 4.48 \times 10^{4}\left(3.06 \times 10^{4}\right) \\ \text { V3 } & \text { N (Nmiss) } & 112(20) & 58(8) & 54(12) \\ & \text { mean (SD) } & 3.66 \times 10^{4}\left(2.65 \times 10^{4}\right) & 2.84 \times 10^{4}\left(1.89 \times 10^{4}\right) & 4.53 \times 10^{4}\left(3.06 \times 10^{4}\right) \\ \Delta(\text { V3-V2) } & N & 111(21) & 58(8) & 53(13) \\ & \text { mean (SD) } & -5.18 \times 10^{3}\left(2.76 \times 10^{4}\right) & -1.03 \times 10^{4}\left(2.36 \times 10^{4}\right) & 3.87 \times 10^{2}\left(3.06 \times 10^{4}\right)\end{array}$

after $\log _{10}$ transformation of values at V2 and V3. The $\log _{10}$ $\Delta \mathrm{V} 3-\mathrm{V} 2[95 \% \mathrm{CI}]$ in the probiotics and placebo groups were $23 \mathrm{ng} / \mathrm{dl}[-57 ; 102]$ and $-137 \mathrm{ng} / \mathrm{dl}[-212 ;-62]$, respectively $(P=0.0044)$. When considered as covariates, breastfeeding history $(P=0.4623)$ or delivery method $(P=0.7670)$ did not influence the results.

\section{Daily stool frequency}

Descriptive statistics for stool frequency are presented in Table 3. Due to strong deviation from assumptions of normality or equal variance (homoscedasticity), $\log _{10}$ transformation was applied. No statistical difference was observed between groups for any week in the ITT population. Analysis on the PP population yielded the same results for statistical significance for most of the 4 week treatment period - week 0, 1, 2 and 3. However, for week 4 , a statistical difference was observed between groups, and increase in the weekly average number of stools/day in the probiotics group $\left(\log _{10} \Delta\right.$ probiotics-placebo [95\%CI] $=0.13[0.01 ; 0.25]$; adjusted $P=0.0334)$.

\section{Faecal microbiota composition}

The abundance of specific taxonomic groups in faecal samples was assessed based on the recommendations from the Chinese Center for Disease Control and Prevention (CCDC) concerning probiotics efficacy for the regulation of intestinal flora composition, and include the changes in Lactobacillus, Bifidobacterium, C. perfringens, Enterococcus, Enterobacter, and Bacteroides counts before and after supplementation. No significant differences in microbiota composition were observed between groups (Table 4). 
Table 3. Statistics on the weekly average number of stools/day for participants in the intent-to-treat population.

\begin{tabular}{|c|c|c|c|c|c|c|}
\hline Week & $\begin{array}{l}\text { Statistical } \\
\text { parameter }^{1}\end{array}$ & All the population $(n=132)$ & Placebo $(n=66)$ & Probiotics $(n=66)$ & $\begin{array}{l}\log _{10} \Delta \text { probiotics-placebo } \\
{[95 \% \mathrm{Cl}]}\end{array}$ & $P$-value \\
\hline \multirow[t]{2}{*}{ Week 0} & $\mathrm{~N}$ (Nmiss) & $113(19)$ & $59(7)$ & $54(12)$ & & \\
\hline & mean (SD) & $1.05(0.602)$ & $1.07(0.645)$ & $1.02(0.556)$ & $0.02[-0.14 ; 0.18]$ & 0.9864 \\
\hline \multirow[t]{2}{*}{ Week 1} & $\mathrm{~N}$ (Nmiss) & $104(28)$ & $55(11)$ & $49(17)$ & & \\
\hline & mean $(S D)$ & $1.15(0.661)$ & $1.13(0.774)$ & $1.17(0.513)$ & $0.05[-0.07 ; 0.17]$ & 0.6828 \\
\hline \multirow[t]{2}{*}{ Week 2} & $\mathrm{~N}$ (Nmiss) & $107(25)$ & $55(11)$ & $52(14)$ & & \\
\hline & mean (SD) & $1.17(0.679)$ & $1.16(0.814)$ & $1.18(0.507)$ & $0.07[-0.07 ; 0.22]$ & 0.5645 \\
\hline \multirow[t]{2}{*}{ Week 3} & $\mathrm{~N}$ (Nmiss) & $107(25)$ & $55(11)$ & $52(14)$ & & \\
\hline & mean $(\mathrm{SD})$ & $1.17(0.579)$ & $1.14(0.591)$ & $1.20(0.570)$ & $0.05[-0.06 ; 0.15]$ & 0.6798 \\
\hline \multirow[t]{2}{*}{ Week 4} & $\mathrm{~N}$ (Nmiss) & $107(25)$ & $54(12)$ & $53(13)$ & & \\
\hline & mean (SD) & $1.05(0.569)$ & $0.95(0.539)$ & $1.14(0.587)$ & $0.10[-0.01 ; 0.21]$ & 0.0943 \\
\hline
\end{tabular}

Table 4. Comparison of changes the abundance of specific taxonomic groups between visit 2 and visit 3 for the placebo and probiotic arms.

\begin{tabular}{|c|c|c|c|}
\hline \multirow[t]{2}{*}{ Species } & \multicolumn{2}{|c|}{ [Variations (V3-V2) \pm SD] ${ }^{1}$ (cfu/g) } & \multirow[t]{2}{*}{$P$-value } \\
\hline & Placebo $(n=66)$ & Probiotic $(n=66)$ & \\
\hline Lactobacillus count & $9.5 \times 10^{9} \pm 4.09 \times 10^{40}$ & $8.68 \times 10^{9} \pm 4.94 \times 10^{10}$ & 0.1465 \\
\hline Bifidobacterium count & $-1.69 \times 10^{10} \pm 2.23 \times 10^{11}$ & $1.11 \times 10^{10} \pm 8.08 \times 10^{10}$ & 0.5567 \\
\hline Enterococcus count & $7.29 \times 10^{9} \pm 4.65 \times 10^{10}$ & $-2.63 \times 10^{9} \pm 8.88 \times 10^{10}$ & 0.2145 \\
\hline Clostridium perfringens count & $-3.93 \times 10^{5} \pm 2.09 \times 10^{6}$ & $-3.64 \times 10^{5} \pm 2.75 \times 10^{6}$ & 0.3466 \\
\hline Enterobacter count & $1.76 \times 10^{10} \pm 8.24 \times 10^{10}$ & $-1.27 \times 10^{10} \pm 6.98 \times 10^{10}$ & 0.0766 \\
\hline Bacteroides count & $-4.44 \times 10^{8} \pm 1.08 \times 10^{10}$ & $6.74 \times 10^{8} \pm 9.27 \times 10^{9}$ & 0.9184 \\
\hline
\end{tabular}

\section{Product safety}

Anthropometric measurements were collected to compare the growth rate between both groups (Table 5). Results showed growth equivalence between groups for weight, height, body mass index, and head circumference $(P>0.05)$. No statistical differences were observed between groups at baseline on the following parameters: age, body mass index, child's gestational age, weight, height, head circumference, and breastfeeding history $(P>0.05)$.

The safety conclusions are based on participants for whom at least one $\mathrm{AE}$ was reported, and who had consumed one of the investigational products $(n=114)$. Among the 114 participants, 106 AEs were notified by the doctors during clinic visits for 52 children. A subgroup of 23 out of 55 (41.82\%) had at least one AE in the probiotics group vs 29 out of 59 (49.15\%) in the placebo group. These proportions were not significantly different between groups $(P=0.4321)$.
For the 23 infants with at least one AE among the 55 babies in the probiotics group, 37 AEs were reported vs 69 AEs for the 29 babies with at least one AE among the 59 babies in the placebo group. The number of AE per baby was significantly lower in the probiotics group $($ mean $=0.67)$ compared to the placebo group $($ mean $=1.17)(P=0.0055)$.

All AEs were declared as mild and unrelated to the product or research. In the probiotics group, the 37 AEs were classified in the following body systems: 21 respiratory, 12 gastrointestinal and 4 dermatological. In the placebo group, the 69 AEs were classified in the following body systems: 38 respiratory, 15 gastro-intestinal and 16 dermatological. None of the AEs were related to the study or study products. No serious AE or effect was reported during the study. Growth rate was normal in both groups, and there was no difference between groups for weight $(P=0.8422)$, height $(P=0.8788)$, body mass index $(P=0.8527)$, or head circumference $(P=0.8827)$. 
Table 5. Anthropometric measurements descriptive statistics of the intent-to-treat population.

\begin{tabular}{|c|c|c|c|c|}
\hline [ $\Delta \mathrm{V} 3-\mathrm{V} 2]$ & Statistics $^{1}$ & All $(n=132)$ & Placebo $(n=66)$ & Probiotic $(n=66)$ \\
\hline & $\mathrm{N}$ (Nmiss) & $115(17)$ & $59(7)$ & $56(10)$ \\
\hline Height (cm) & mean (SD) & $1.6(1.16)$ & $1.5(1.20)$ & $1.8(1.11)$ \\
\hline Weight $(\mathrm{g}) \mathrm{C}$ & mean (SD) & $318.5(265.13)$ & $302.9(234.18)$ & $335.0(295.51)$ \\
\hline Body mass index $\left(\mathrm{kg} / \mathrm{m}^{2}\right)$ & mean (SD) & $-0.2(0.77)$ & $-0.1(0.77)$ & $-0.2(0.78)$ \\
\hline Head circumference $(\mathrm{cm})$ & mean (SD) & $0.6(0.63)$ & $0.7(0.72)$ & $0.6(0.52)$ \\
\hline
\end{tabular}

\section{Discussion and conclusions}

In this study, we show that probiotics administration can prevent the decrease of faecal SIgA levels that is normally observed in formula-fed infants (Bakker-Zierikzee et al., 2006). While absolute SIgA levels are higher at any time point in breastfed infants vs those fed on formula, (BakkerZierikzee et al., 2006) previous studies have shown that intestinal SIgA levels decrease over time in both breastfed and formula-fed infants (Bakker-Zierikzee et al., 2006). Under normal conditions, SIgA levels increase from birth until the first month of life, then start decreasing until they reach a stable level at around 6 months (Maruyama et al., 2009). Lower SIgA levels in the gastrointestinal tract of newborns were associated with a higher incidence of infectious diseases (Wold and Adlerberth, 2000). Consequently, faecal SIgA levels, which have been shown to be representative of SIgA concentrations in the colon, are used as a surrogate outcome measure for immune competence in infants (Scholtens et al., 2008). Indeed, faecal SIgA levels represent a good surrogate endpoint for immunity development because of the resistance of this protein to intraluminal proteolysis and its inability to directly activate complement or inflammatory responses (Scholtens et al., 2008). Hence, in our study, the higher faecal SIgA level found in the probiotics group at the end of treatment visit (V3) may indicate that the probioticsupplemented formula supported a higher SIgA production over time, thereby enhancing mucosal immunity and protection against pathogens compared to the placebosupplemented milk formula.

Several confounding factors that could possibly affect participants' SIgA levels were considered. First, all the infants included in our study were vaccinated in accordance with the guidelines of the Chinese national vaccination program; consistently there was no difference related to vaccination between two groups. The frequency and seriousness of diseases, like vomiting, diarrhoea, fever and the defecation characteristics were recorded in the infant diaries and showed no difference between groups during the study. Therefore, it is justified to consider that the effects of variables such as vaccination, disease severity, and defecation characteristics on SIgA production were minimised in our study. As SIgA levels are substantially higher in breastfed infants than in formula-fed infants, (Bakker-Zierikzee et al., 2006) we excluded breastfed babies from this study; our goal was to assess the potential benefits of probiotic supplementation in formula-fed babies specifically. Furthermore, we did not expect probiotics alone to upregulate SIgA levels to a level comparable as that observed in breastfed babies, especially with a formula containing minimal levels of prebiotic oligosaccharides (see exclusion criteria). Indeed, recent studies have suggested that prebiotic-probiotic combinations (e.g. synbiotics) could efficiently promote immunity in formula-fed infants (Chen et al., 2007; Vandenplas et al., 2017). It is likely that specific combinations of prebiotics and probiotic strains will harbour different efficiencies in regard to that outcome, and the notion of 'ideal pairing' should be taken into consideration when designing future studies.

Our findings are consistent with previous studies about the role of probiotics (with or without prebiotics) in children from different age groups. A study showed that salivary IgA levels were elevated in the probiotic groups, and concluded that the tested probiotics were effective at inducing an intestinal immune response while improving and maintaining immunity (Chen et al., 2007). In our study, the levels of salivary IgA showed an increasing trend in the probiotic-supplemented infants, but without reaching significance in the current conditions tested (i.e. 4-week treatment, minimal level of prebiotics). Another trial also showed that probiotics, in the presence of nondigestible oligosaccharides, could decrease the occurrence of seasonal winter diseases and reduce the loss of school days in children (Cazzola et al., 2010a). Furthermore, two recent trials revealed that, individually, the probiotic strains composing the formulation studied herein were safe to consume in infants aged between 3 and 12 months, and could modulate gut microbiota composition and cytokine levels in this population following an 8 week treatment period (De Andres et al., 2018; Manzano et al., 2017). In addition, existing evidence suggest that $B$. infantis can 
secrete biologically active peptides affecting epithelial and immune cells, playing a role in maintaining intestinal epithelial cell barrier integrity through the regulation of tight junction proteins and the mitogen-activated protein kinase (MAPK) pathway (Ewaschuk et al., 2008). Moreover, the probiotic formulation used herein could modulate Th1 or Th2 immune responses in Wistar rats (Cazzola et al., 2010b) or human intestinal epithelial cells (MacPherson et al., 2014).

In our study, cytokine levels were not assessed. However, previous studies have suggested cytokines as possible mediators of the effects of the investigated probiotics on SIgA levels and immune responses. Treatment with heat-treated L. helveticus R0052 was shown to induce the proliferation of mouse splenocytes in vitro, and to increase total levels of non-antigen specific polyclonal antibodies (Easo et al., 2002). Furthermore, other probiotic strains, namely Lactobacillus GG and Bifidobacterium lactis $\mathrm{Bb}-12$, could enhance IgA responses in cow-milk fed infants, although a mechanism for the action of these strains could not be formally identified (Rautava et al., 2006). Over time, evidence supporting the ability of several probiotic strains, and particularly Lactobacillus species, at modulating immune responses in various contexts has accumulated. The general mechanisms are thought to involve the modulation of Toll-like receptors signalling and cytokine production. For instance, the same probiotic formulation as used in this study was shown to attenuate the polyI:C-induced activation of the nuclear factor kappa beta signalling cascade downstream of Toll-like receptor-3 in HT-29 cells (MacPherson et al., 2014, 2017). Interestingly, in this particular in vitro system, the threestrain formulation was more efficient than each strain used individually, which is in accordance with the notion that multistrain probiotic products comprising complementary strains may be more efficient (MacPherson et al., 2017). More recently, Pediococcus acidilactici K15 was shown to enhance SIgA production in vitro using primary peripheral blood mononuclear cells, which occurred in an interleukin (IL)-6- or IL-10-dependent manner (Kawashima et al., 2018). This group proposed that cytokines produced by dendritic cells could be contributing at enhancing SIgA at mucosal site in humans. A consensus appears to exist around the role of certain probiotic strains, but not others, at enhancing mucosal immunity. Studies investigating the potential molecular mechanisms involved have generated variable results that, arguably, may depend on the compartmentalisation of immune signalling along the intestinal tract in vivo, or on the cellular model system and experimental conditions used. Indeed, an array of cytokines were reported to regulate SIgA production in the intestines, including tumour growth factor (TGF)- $\beta$, IL-4, IL-5, IL-6, IL-10 and IL-21 (Mantis et al., 2011). Furthermore, TGF- $\beta$ and IL-10 were also associated with maintenance of mucosal tolerance (Mantis et al., 2011). In light of the current knowledge base, the characterisation of specific molecular mechanisms and cell types involved in the maintenance of SIgA levels in the intestine of healthy, non-immune challenged, formula-fed infants warrants further study.

With respect to the microflora diversity in infants, a group of researchers have studied the impact of feeding patterns on the intestinal microflora in 3-6 months healthy Chinese infants. They found that the bifidobacteria count in the faeces of breastfed infants was more than five times the count found in formula-fed infants (6.63 vs $1.29 \%$ ). The Lactobacillus count was also higher in breastfed infants compared with formula-fed infants (5.798 vs 4.12\%), although to a lesser degree (Fan et al., 2013). This is indicative of the importance of Bifidobacterium presence in the intestines of infants with an immature immune system. However, since our microbiota composition analyses required the comparison of two groups receiving a type of supplemented formula (probiotics or placebo) containing minimal prebiotics amounts, as opposed to comparing more distinct groups (i.e. formula-fed vs breastfed infants), we were unable to detect differences in the changes of microbiota composition between groups after four weeks of treatment (Table 4).

To conclude, the present randomised, double-blinded study results show that probiotics may help maintain faecal SIgA levels in formula-fed infants, suggesting that it is possible to stimulate the development of the mucosal immune response in formula-fed infants with the appropriate probiotic supplement. In addition, it is clear from our results that the probiotic formulation used in this study is well tolerated and safe in 3-6 months old infants and could enhance mucosal immunity in healthy formula-fed infants. Although evidence is accumulating to support the role of probiotics in immune system function and development, the impact of microbiota establishment and the role of probiotics on the development of mucosal immunity in infants remains unclear and needs to be elucidated in order to develop milk formulas that will ultimately achieve the same level of health benefits as breastmilk.

\section{Conflict of interest}

L. Xiao, C. Gong, Y. Ding, and G. Ding declare no conflict of interest. X. Xu is employed by Lallemand Health Solutions, a company that manufactures probiotics for human health applications. C. Deng, X. Ze, and P. Malard are employed by Biostime, an $\mathrm{H} \& \mathrm{H}$ group company, that commercializes children's probiotic formulations and infant milk formula. $\mathrm{X}$. Ben received fees from Biostime for conducting this clinical trial. 


\section{References}

Aloisio, I., Prodam, F., Giglione, E., Bozzi Cionci, N., Solito, A., Bellone, S., Baffoni, L., Mogna, L., Pane, M., Bona, G. and Di Gioia, D., 2018. Three-month feeding integration with Bifidobacterium strains prevents gastrointestinal symptoms in healthy newborns. Frontiers in Nutrition 5: 39-39. DOI: https://doi.org/10.3389/fnut.2018.00039

Bakker-Zierikzee, A., Tol, E., Kroes, H., Alles, M., Kok, F. and Bindels, J., 2006. Faecal SIgA secretion in infants fed on pre- or probiotic infant formula. Pediatric Allergy and Immunology 17: 134-140.

Booth, C.K., Dwyer, D.B., Pacque, P.F. and Ball, M.J., 2009. Measurement of immunoglobulin A in saliva by particle-enhanced nephelometric immunoassay: sample collection, limits of quantitation, precision, stability and reference range. Annals of Clinical Biochemistry 46: 401-406. DOI: https://doi.org/10.1258/acb.2009.008248

Cacho, N.T. and Lawrence, R.M., 2017. Innate immunity and breast milk. Frontiers in Immunology 8: 584. DOI: https://doi.org/10.3389/ fimmu.2017.00584

Cazzola, M., Pham-Thi, N., Kerihuel, J.C., Durand, H. and Bohbot, S., 2010a. Efficacy of a synbiotic supplementation in the prevention of common winter diseases in children: a randomized, double-blind, placebo-controlled pilot study. Therapeutic Advances in Respiratory Disease 4: 271-278. DOI: https://doi.org/10.1177/1753465810379010

Cazzola, M., Tompkins, T.A. and Matera, M.G., $2010 \mathrm{~b}$. Immunomodulatory impact of a synbiotic in $\mathrm{T}(\mathrm{h}) 1$ and $\mathrm{T}(\mathrm{h}) 2$ models of infection. Therapeutic Advances in Respiratory Disease 4: 259270. DOI: https://doi.org/10.1177/1753465810379009

Chen, L., Ouyang, J., Liao, W. and Zhang, W., 2007. Effect of synbiotics probiotics on improving mucosal immunity in children's digestive tract. Chinese Journal of Microecology 2: 137-141. [in Chinese]

De Andres, J., Manzano, S., Garcia, C., Rodriguez, J.M., EspinosaMartos, I. and Jimenez, E., 2018. Modulatory effect of three probiotic strains on infants' gut microbial composition and immunological parameters on a placebo-controlled, double-blind, randomised study. Beneficial Microbes 9: 573-584. DOI: https://doi.org/10.3920/ bm2017.0132

Deshpande, G., Jape, G., Rao, S. and Patole, S., 2017. Benefits of probiotics in preterm neonates in low-income and medium-income countries: a systematic review of randomised controlled trials. BMJ 7: e017638. DOI: https://doi.org/10.1136/bmjopen-2017-017638

Dion, C., Montagne, P., Bene, M.C. and Faure, G., 2004. Measurement of faecal immunoglobulin a levels in young children. Journal of Clinical Laboratory Analysis 18: 195-199. DOI: https://doi. org/10.1002/jcla.20022

Easo, J.G., Measham, J.D, Munroe, J. and Green-Johnson, J., 2002. Immunostimulatory actions of lactobacilli: mitogenic induction of antibody production and spleen cell proliferation by Lactobacillus delbrueckii subsp. bulgaricus and Lactobacillus acidophilus. Food and Agricultural Immunology 14: 73-83. DOI: https://doi. org/10.1080/09540100220137682

Ewaschuk, J.B., Diaz, H., Meddings, L., Diederichs, B., Dmytrash, A., Backer, J., Looijer-van Langen, M. and Madsen, K.L., 2008. Secreted bioactive factors from Bifidobacterium infantis enhance epithelial cell barrier function. American Journal of Physiology Gastrointestinal and Liver Physiology 295: G1025-G1034. DOI: https://doi.org/10.1152/ajpgi.90227.2008
Fan, W., Huo, G., Li, X., Yang, L., Duan, C., Wang, T. and Chen, J., 2013. Diversity of the intestinal microbiota in different patterns of feeding infants by Illumina high-throughput sequencing. World Journal of Microbiology and Biotechnology 29: 2365-2372. DOI: https://doi.org/10.1007/s11274-013-1404-3

Gensollen, T., Iyer, S.S., Kasper, D.L. and Blumberg, R.S., 2016. How colonization by microbiota in early life shapes the immune system. Science 352: 539-544. DOI: https://doi.org/10.1126/science.aad9378 Giovannini, M., Verduci, E., Gregori, D., Ballali, S., Soldi, S., Ghisleni, D., Riva, E. and Group P.T.S., 2014. Prebiotic effect of an infant formula supplemented with galacto-oligosaccharides: randomized multicenter trial. Journal of the American College of Nutrition 33: 385-393. DOI: https://doi.org/10.1080/07315724.2013.878232 Guaraldi, F. and Salvatori, G., 2012. Effect of breast and formula feeding on gut microbiota shaping in newborns. Frontiers in Cellular and Infection Microbiology 2: 94. DOI: https://doi.org/10.3389/ fcimb.2012.00094

Jacobs, S.E., Tobin, J.M., Opie, G.F., Donath, S., Tabrizi, S.N., Pirotta, M., Morley, C.J. and Garland, S.M., 2013. Probiotic effects on late-onset sepsis in very preterm infants: a randomized controlled trial. Pediatrics 132: 1055-1062. DOI: https://doi.org/10.1542/ peds.2013-1339

Jakaitis, B.M. and Denning, P.W., 2014. Human breast milk and the gastrointestinal innate immune system. Clinics in Perinatology 41: 423-435. DOI: https://doi.org/10.1016/j.clp.2014.02.011

Kato, L.M., Kawamoto, S., Maruya, M. and Fagarasan, S., 2014. The role of the adaptive immune system in regulation of gut microbiota. Immunological Reviews 260: 67-75. DOI: https://doi.org/10.1111/ imr. 12185

Kawashima, T., Ikari, N., Kouchi, T., Kowatari, Y., Kubota, Y., Shimojo, N. and Tsuji, N.M., 2018. The molecular mechanism for activating IgA production by Pediococcus acidilactici $\mathrm{K} 15$ and the clinical impact in a randomized trial. Scientific Reports 8: 5065. DOI: https://doi.org/10.1038/s41598-018-23404-4.

Lee, S.A., Lim, J.Y., Kim, B.-S., Cho, S.J., Kim, N.Y., Kim, O.B. and Kim, Y., 2015. Comparison of the gut microbiota profile in breast-fed and formula-fed Korean infants using pyrosequencing. Nutrition Research and Practice 9: 242-248. DOI: https://doi.org/10.4162/ nrp.2015.9.3.242

Lewis, D.B. and Wilson, C.B., 2011. Developmental immunology and role of host defenses in fetal and neonatal susceptibility to infection. Infectious diseases of the fetus and newborn. Elsevier, New York, NY, USA, pp. 80-191. DOI: https://doi.org/10.1016/b978-1-41606400-8.00004-3

MacPherson, C., Audy, J., Mathieu, O. and Tompkins, T.A., 2014. Multistrain probiotic modulation of intestinal epithelial cells' immune response to a double-stranded RNA ligand, poly(i.c). Applied and Environmental Microbiology 80: 1692-1700. DOI: https://doi.org/10.1128/aem.03411-13

MacPherson, C.W., Shastri, P., Mathieu, O., Tompkins, T.A. and Burguiere, P., 2017. Genome-wide immune modulation of TLR3mediated inflammation in intestinal epithelial cells differs between single and multi-strain probiotic combination. PLoS ONE 12: e0169847. DOI: https://doi.org/10.1371/journal.pone.0169847 
Mantis, N.J., Rol, N. and Corthesy, B., 2011. Secretory IgA's complex roles in immunity and mucosal homeostasis in the gut. Mucosal Immunology 4: 603-611. DOI: https://doi.org/10.1038/mi.2011.41 Manzano, S., De Andres, J., Castro, I., Rodriguez, J.M., Jimenez, E. and Espinosa-Martos, I., 2017. Safety and tolerance of three probiotic strains in healthy infants: a multi-centre randomized, double-blind, placebo-controlled trial. Beneficial Microbes 8: 569-578. DOI: https://doi.org/10.3920/bm2017.0009

Maruyama, K., Hida, M., Kohgo, T. and Fukunaga, Y., 2009. Changes in salivary and fecal secretory IgA in infants under different feeding regimens. Pediatrics International 51: 342-345. DOI: https://doi. org/10.1111/j.1442-200X.2008.02748.x

Penders, J., Vink, C., Driessen, C., London, N., Thijs, C. and Stobberingh, E.E., 2005. Quantification of Bifidobacterium spp., Escherichia coli and Clostridium difficile in faecal samples of breast-fed and formula-fed infants by real-time PCR. FEMS Microbiology Letters 243: 141-147. DOI: https://doi.org/10.1016/j. femsle.2004.11.052

Rautava, S., Arvilommi, H. and Isolauri, E., 2006. Specific probiotics in enhancing maturation of IgA responses in formula-fed infants. Pediatric Research 60: 221-224. DOI: https://doi.org/10.1203/01. pdr.0000228317.72933.db

Rodríguez, J.M., Murphy, K., Stanton, C., Ross, R.P., Kober, O.I., Juge, N., Avershina, E., Rudi, K., Narbad, A., Jenmalm, M.C., Marchesi, J.R. and Collado, M.C., 2015. The composition of the gut microbiota throughout life, with an emphasis on early life. Microbial Ecology in Health and Disease 26: 26050-26050. DOI: https://doi.org/10.3402/ mehd.v26.26050

Scholtens, P.A., Alliet, P., Raes, M., Alles, M.S., Kroes, H., Boehm, G., Knippels, L.M., Knol, J. and Vandenplas, Y., 2008. Fecal secretory immunoglobulin A is increased in healthy infants who receive a formula with short-chain galacto-oligosaccharides and long-chain fructo-oligosaccharides. Journal of Nutrition 138: 1141-1147. DOI: https://doi.org/10.1093/jn/138.6.1141

Simeoni, U., Berger, B., Junick, J., Blaut, M., Pecquet, S., Rezzonico, E., Grathwohl, D., Sprenger, N., Brussow, H., Study, T., Szajewska, H., Bartoli, J.M., Brevaut-Malaty, V., Borszewska-Kornacka, M., Feleszko, W., Francois, P., Gire, C., Leclaire, M., Maurin, J.M., Schmidt, S., Skorka, A., Squizzaro, C. and Verdot, J.J., 2016. Gut microbiota analysis reveals a marked shift to bifidobacteria by a starter infant formula containing a synbiotic of bovine milk-derived oligosaccharides and Bifidobacterium animalis subsp. lactis CNCM I-3446. Environmental Microbiology 18: 2185-2195. DOI: https:// doi.org/10.1111/1462-2920.13144

Skorka, A., Piescik-Lech, M., Kolodziej, M. and Szajewska, H., 2017. To add or not to add probiotics to infant formulae? An updated systematic review. Beneficial Microbes 8: 717-725. DOI: https:// doi.org/10.3920/bm2016.0233

Stojkovic, A., Simovic, A., Bogdanovic, Z., Bankovic, D. and Poskurica, M., 2016. Clinical trial/experimental study (consort compliant): optimal time period to achieve the effects on synbiotic-controlled wheezing and respiratory infections in young children. Srpski Arhiv za Celokupno Lekarstvo 144: 38-45.
Thomas, J.P., Raine, T., Reddy, S. and Belteki, G., 2017. Probiotics for the prevention of necrotising enterocolitis in very low-birth-weight infants: a meta-analysis and systematic review. Acta Paediatrica 106: 1729-1741. DOI: https://doi.org/10.1111/apa.13902

Tzira, D., Prezerakou, A., Papadatos, I., Vintila, A., Bartzeliotou, A., Apostolakou, F., Papassotiriou, I. and Papaevangelou, V., 2018. Salivary biomarkers may measure stress responses in critically ill children. SAGE Open Medicine 6: 2050312118802452. DOI: https:// doi.org/10.1177/2050312118802452

Vandenplas, Y., Analitis, A., Tziouvara, C., Kountzoglou, A., Drakou, A., Tsouvalas, M., Mavroudi, A. and Xinias, I., 2017. Safety of a new synbiotic starter formula. Pediatric Gastroenterology, Hepatology and Nutrition 20: 167-177. DOI: https://doi.org/10.5223/ pghn.2017.20.3.167

Wang, M., Li, M., Wu, S., Lebrilla, C.B., Chapkin, R.S., Ivanov, I. and Donovan, S.M., 2015. Fecal microbiota composition of breast-fed infants is correlated with human milk oligosaccharides consumed. Journal of Pediatric Gastroenterology and Nutrition 60: 825-833. DOI: https://doi.org/10.1097/MPG.0000000000000752

World Health Organisation (WHO), 2018a. Exclusive breastfeeding for optimal growth, development and health of infants. e-Library of Evidence for Nutrition Actions (eLENA). Available at: https:// www.who.int/elena/titles/exclusive_breastfeeding/en/

World Health Organisation (WHO), 2018b. Preterm birth fact sheet. Available at: https://www.who.int/en/news-room/fact-sheets/detail/ preterm-birth

Wold, A.E. and Adlerberth, I., 2000. Breast feeding and the intestinal microflora of the infant - implications for protection against infectious diseases. Advances in Experimental Medicine and Biology 478: 77-93. DOI: https://doi.org/10.1007/0-306-46830-1_7

Wopereis, H., Oozeer, R., Knipping, K., Belzer, C. and Knol, J., 2014. The first thousand days - intestinal microbiology of early life: establishing a symbiosis. Pediatric Allergy and Immunology 25: 428-438. DOI: https://doi.org/10.1111/pai.12232

Xiao, L., Ding, G., Ding, Y., Deng, C., Ze, X., Chen, L., Zhang, Y., Song, L., Yan, H., Liu, F. and Ben, X., 2017. Effect of probiotics on digestibility and immunity in infants: a study protocol for a randomized controlled trial. Medicine 96: e5953. DOI: https://doi. org/10.1097/md.0000000000005953

Yatsunenko, T., Rey, F.E., Manary, M.J., Trehan, I., Dominguez-Bello, M.G., Contreras, M., Magris, M., Hidalgo, G., Baldassano, R.N., Anokhin, A.P., Heath, A.C., Warner, B., Reeder, J., Kuczynski, J., Caporaso, J.G., Lozupone, C.A., Lauber, C., Clemente, J.C., Knights, D., Knight, R. and Gordon, J.I., 2012. Human gut microbiome viewed across age and geography. Nature 486: 222-227. DOI: https://doi. org/10.1038/nature11053

Zhang, G.-Q., Hu, H.-J., Liu, C.-Y., Shakya, S. and Li, Z.-Y., 2016. Probiotics for preventing late-onset sepsis in preterm neonates: a PRISMA-compliant systematic review and meta-analysis of randomized controlled trials. Medicine 95: e2581-e2581. DOI: https://doi.org/10.1097/MD.0000000000002581 
